\title{
Deuterium Retention Properties of Co-Deposited Carbon Films Produced at Wall Gaps ${ }^{*)}$
}

\author{
Yuji NOBUTA, Jun KANAZAWA, Yuji YAMAUCHI, Tomoaki HINO, Kenji YOKOYAMA ${ }^{1)}$, \\ Satoshi SUZUKI ${ }^{1)}$, Koichiro EZATO ${ }^{1)}$, Mikio ENOEDA ${ }^{1)}$, Masato AKIBA ${ }^{1)}$, \\ Satoshi AKAMARU ${ }^{2)}$ and Yuji HATANO ${ }^{2)}$ \\ Laboratory of Plasma Physics and Engineering, Hokkaido University, Sapporo 060-8628, Japan \\ 1) Japan Atomic Energy Agency, 801-1 Mukouyama, Naka-shi, Ibaraki 311-0193, Japan \\ ${ }^{2)}$ Hydrogen Isotope Research Center, University of Toyama, 3190 Gofuku, Toyama 930-8555, Japan
}

(Received 22 November 2012 / Accepted 13 November 2013)

\begin{abstract}
Deuterium retention properties in co-deposited carbon film produced in gap and the relationship between this retention behavior and the crystal structure of carbon film were investigated. In the case of a wide gap, the atomic ratio of deuterium to carbon $(\mathrm{D} / \mathrm{C})$ in the film was almost constant at any depth in the gap, while in the case of a narrow gap the $\mathrm{D} / \mathrm{C}$ ratio decreased with increasing distance from the gap entrance. The micro structure of carbon film tended to be more amorphous for the film produced at locations deeper in the gap. Thermal desorption spectra of $\mathrm{D}_{2}$ in the film produced near the gap entrance showed one broad main peak at around $1100 \mathrm{~K}$, while that in the film produced near the bottom showed very sharp peaks at around $950 \mathrm{~K}$. This difference in desorption behavior was related with the differences of micro structure.
\end{abstract}

(c) 2013 The Japan Society of Plasma Science and Nuclear Fusion Research

Keywords: carbon, co-deposition, gap, deuterium retention, TDS, desorption

DOI: $10.1585 /$ pfr.8.2405174

\section{Introduction}

From the point of view of safety and operational scheduling, estimating in-vessel tritium retention is a main issue for ITER and next step fusion devices. Carbon-based materials have been widely used as plasma-facing materials in fusion devices and are candidates for the ITER divertor target material because of their low atomic number and good thermal properties. A carbon-based material is easily sputtered by bombardment of incident hydrogen particles and sputtered carbon is redeposited on the walls incorporating fuel hydrogen, which leads to the formation of a hydrogenated carbon film (co-deposited carbon film). It is reported that almost all the hydrogen isotopes remaining in the vacuum vessel accumulated on the co-deposited carbon film [1]. On the plasma facing surface, there are many openings with various widths between the plasmafacing tiles/components, which are generally called the 'gap'. Sputtered carbon particles, especially neutral particles, could enter into the gap and are deposited on the side surface in the gap. Therefore, the co-deposition occurs not only on plasma-facing surfaces but also in the gaps. Since removal of the fuel hydrogen accumulated in co-deposited carbon film in the gap is quite difficult [2-4], the co-deposition of hydrogen with carbon in the gap is a significant problem. In order to evaluate the in-vessel tritium inventory, it is important to investigate fuel hydrogen

author's e-mail: y-nobuta@eng.hokudai.ac.jp

*) This article is based on the presentation at the 22nd International Toki Conference (ITC22). retention of the co-deposited carbon film in the gap.

Carbon atoms and hydrocarbons are emitted from the carbon material surface by physical and chemical sputtering processes, respectively, part of which is ionized and/or dissociated in the plasma. For hydrogen, atoms and ions enter the gap. Therefore, many types of carbon and hydrogen particles can enter the gap and deposit there, which is a very complicated process. A separate investigation of the deposition process for each case is needed. Furthermore, deposition of neutral particles would be more important than that of ions because neutral particles can enter more deeply into the gap in magnetic confinement fusion devices.

In this study, the co-deposition of deuterium with carbon atoms in the gap with different gap widths was prepared by using a deuterium arc discharge with carbon electrodes. The carbon deposition behavior in the gap and the deuterium retention properties in the co-deposited carbon film were investigated. The relationship between deuterium desorption behavior and the microstructure of the film is also discussed.

\section{Experimental}

Co-deposited carbon film used in this study was prepared by using a deuterium arc discharge. Figure 1 shows a schematic diagram of the vacuum chamber used for the preparation of co-deposited carbon film. The deuterium arc discharge was conducted with a discharge current of 


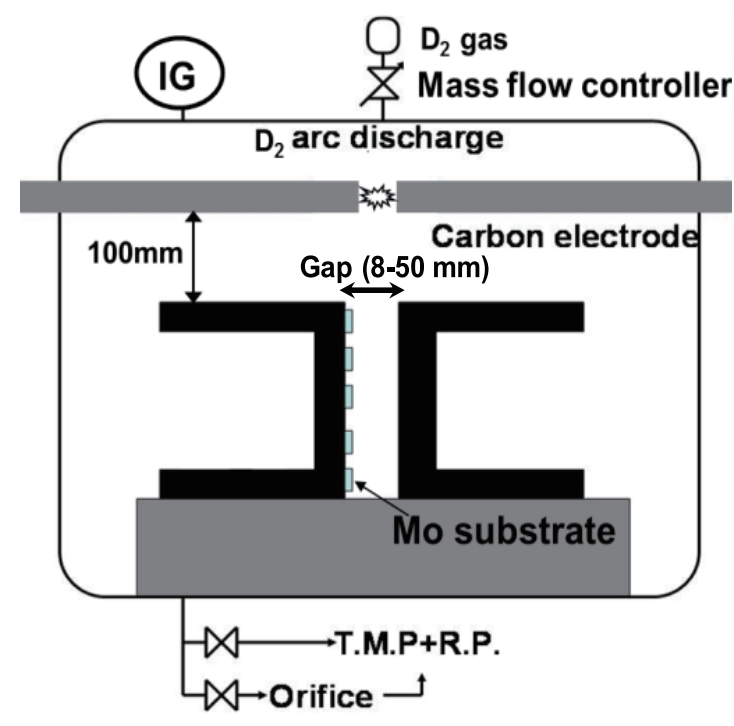

Fig. 1 Schematic diagram of arc discharge chamber for preparing co-deposited carbon film.

$50 \mathrm{~A}$ and a voltage of $11-16 \mathrm{~V}$, respectively. During the arc discharge, a large electric current flowed through a very small area on the electrode surface, leading to sublimation of carbon. Deuterium atoms were generated through dissociation of deuterium molecules in the arc discharge plasma. The sample stage with a gap width of $8-50 \mathrm{~mm}$ and gap depth of $100 \mathrm{~mm}$ was set just below the arc discharge plasma.

The discharge pressure was kept at $7 \pm 2 \mathrm{~Pa}$. During the discharge, both carbon and deuterium atoms were deposited on Mo substrates mounted on the sides of the gap. The deposition rate in each case was $2-4 \mathrm{~nm} / \mathrm{s}$ for the samples placed $10 \mathrm{~mm}$ away from the gap entrance, and decreased as the distance from the gap entrance increased. The substrate temperature measured by using thermocouples directory attached to samples during the discharge were kept at $573 \mathrm{~K}$. After carbon deposition, the samples were extracted from the chamber, and deuterium retention and desorption behavior of the co-deposited carbon film were investigated with thermal desorption spectroscopy (TDS). During the TDS analysis, the samples were heated at a rate of $0.5 \mathrm{~K} / \mathrm{s}$ up to $1273 \mathrm{~K}$ and then kept at $1273 \mathrm{~K}$ for $20 \mathrm{~min}$. The crystal structure of the carbon film was examined using Raman spectroscopy.

\section{Results and Discussion}

The amount of carbon deposited in the gap decreased roughly exponentially with increasing distance from the gap entrance. Figure 2 shows the dependence of the amount of deposited carbon on $\sin \theta / r^{2}$, where $r$ is the distance from the point of origin to the place where the carbon atom deposits and $\theta$ an inclination direction of incident carbon atom against the gap side surface (see inset of Fig. $\dot{2}$ ). The volume of the arc discharge plasma was very small and

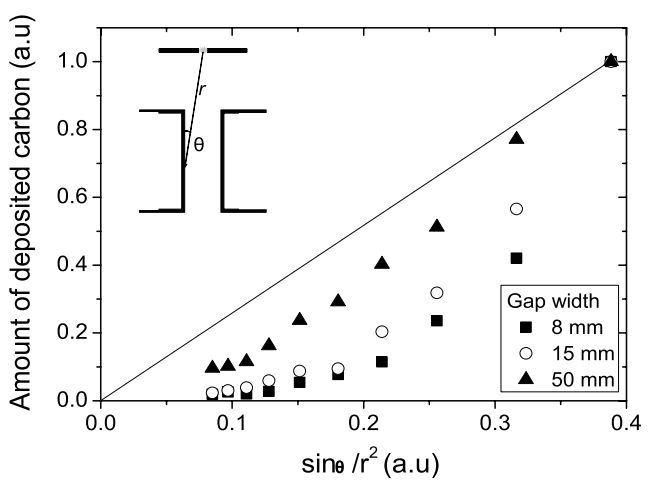

Fig. 2 Dependence of amount of deposited carbon in gap and $\sin \theta / r^{2}$ (see inset and text).

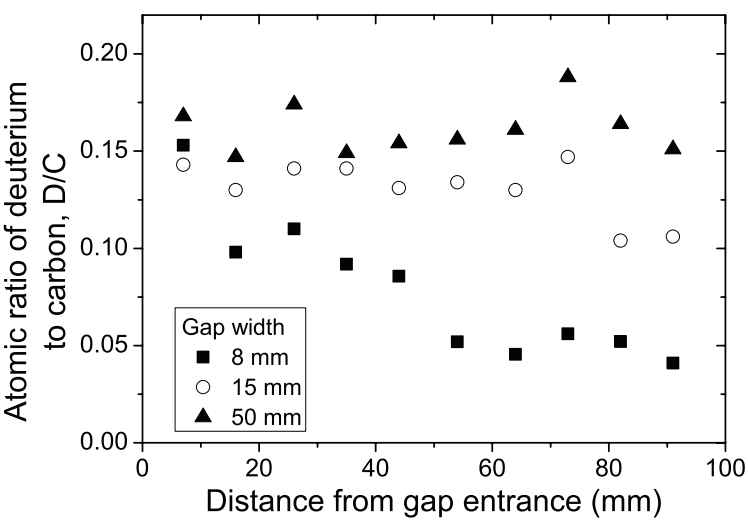

Fig. 3 Dependence of atomic ratio of deuterium to carbon (D/C) on distance from gap entrance.

sublimation of carbon mainly occurred in a very narrow area on the cathode surface. Therefore, the source of carbon emission can be regarded as a point source and carbon atom was thought to be emitted in the random direction from the source. Carbon atom has a high sticking probability because of its high reactivity [5]. Then, once carbon atom entering the gap impinges the gap side surface, the atom should deposit there. In a situation where carbon atom doesn't collide with deuterium molecules until they impinge onto gap side surface, a carbon atom flux onto the surface, in general, is proportional to $\sin \theta / r^{2}$. In this study, as seen in Fig. 2, the proportional connection wasn't observed. Actually, a mean free pass of carbon atom in this experimental condition was estimated to be approximately $1 \mathrm{~mm}$, which was shorter than the gap depth $(10 \mathrm{~cm})$. This indicates that some carbon atoms emitted from the source should collide with the ambient deuterium molecules. In a previous study of the authors, it has been found that the collision with ambient molecules is an important factor for carbon deposition profile in gap [6].

Figure 3 shows the dependence of the atomic ratio of deuterium to carbon on the distance from the gap entrance. The number of atoms of deuterium and carbon in the film were estimated using a TDS technique and by measuring 
(a)

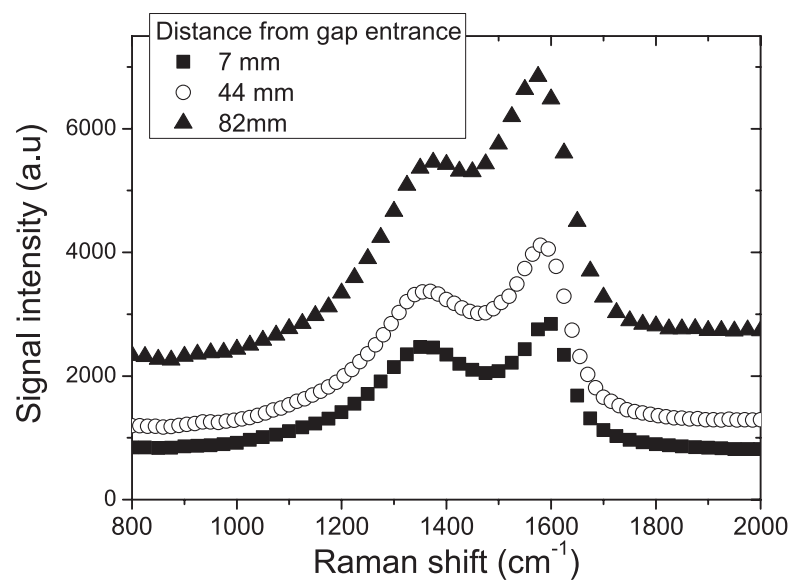

(b)

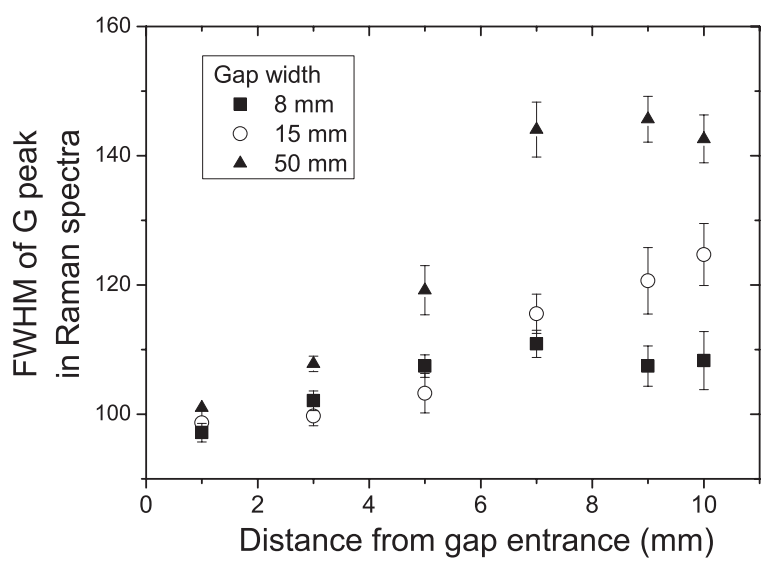

Fig. 4 Raman spectra of the carbon films produced with gap width of $50 \mathrm{~mm}$ (a) and dependence of a full width at half maximum of G-peak (at $1580 \mathrm{~cm}^{-1}$ ) of Raman spectra on distance from gap entrance (b).

the weight gain of substrates after carbon deposition, respectively. The $\mathrm{D} / \mathrm{C}$ ratio in the carbon film produced near the entrance of the gap was almost the same for all cases. In the case of a wide gap $(50 \mathrm{~mm}), \mathrm{D} / \mathrm{C}$ was almost constant in the gap, while in the case of a narrow gap $(8 \mathrm{~mm}), \mathrm{D} / \mathrm{C}$ decreased with increasing distance from the gap entrance. As mentioned above, some carbon and deuterium atoms entering the gap collide with ambient deuterium molecules and then alter their direction of travel. In a collision of two particles, a magnitude correlation of atomic mass between two collisional particles influences the change of direction of the travel after collision. In the collision with a deuterium molecule, the degree of the change of the direction in carbon atom would smaller than that in the case of deuterium atom because of a larger mass of carbon atom against deuterium molecule. On the other hand, a deuterium atom, which is lighter than a deuterium molecule, could significantly alter the direction of travel by collisions with deuterium molecules and then easily impinge on the gap side surface. Therefore, deuterium atoms had a lower possibility of reaching deep into the gap compared to carbon atoms, especially in the narrow gap case. This might be a possible reason for the reduction of the $\mathrm{D} / \mathrm{C}$ in the narrow gap case.

Figure 4 (a) shows the Raman spectra in the carbon film in the case of a $50 \mathrm{~mm}$ gap width. The spectra showed two main peaks at around $1360 \mathrm{~cm}^{-1}$ and $1580 \mathrm{~cm}^{-1}$, which are generally called the D-peak and G-peak, respectively. It has been found that the G-peak and D-peak are due to stretching vibrations of the carbon double bond and its conjugated forms, and the vibration characteristics of the benzene skeleton, respectively, and that the increase in a G-peak full width at half maximum (FWHM) is considered to originate from the increase in disorder in bond length and angle [7,8]. Figure 4(b) shows the relationship of a G-peak FWHM with the distance from the gap entrance. For carbon films produced near the gap entrance, the FWHM became almost the same value for all cases. This indicates that the gap width have no effect on microstructure of carbon film produced close to gap entrance. The G-peak FWHM increased with distance from the gap entrance indicates that the crystal structure of the carbon film became more disordered structure (amorphous) in the carbon film produced in deeper regions.

Carbon atom has, again, a high sticking probability because of its high reactivity. Then, once carbon atom entering the gap impinges the gap side surface, the atom should deposit there. Therefore, the carbon atoms, which experienced no collision with the gap side surface, could reach in deeper regions. Carbon atoms generated by sublimation at the carbon electrode surface lose energy through collision with deuterium molecules until carbon atoms finally deposit on the gap side surface. In the case of carbon deposition with lower incident energy, the crystal structure of carbon film tends to be a more amorphous structure $[9,10]$. The difference of incident energy of carbon atoms at each place in the gap would be responsible for differences in the crystal structure. For carbon film produced with a narrow gap $(8 \mathrm{~mm})$, the FWHM in deeper region became smaller compared with the wide gap $(50 \mathrm{~mm})$ case. It has been reported that carbon films with higher hydrogen concentration tend to be more amorphous [11]. As seen in Fig. 2, the D/C of the film produced with wide gap width $(50 \mathrm{~mm})$ was almost constant while the $\mathrm{D} / \mathrm{C}$ of the narrow gap case $(8 \mathrm{~mm})$ decreased with increasing distance from the gap entrance. This decrease of deuterium concentration $(\mathrm{D} / \mathrm{C})$ in the film produced with narrow gap might also be a reason for differences in the micro structure.

Figure 5 shows the thermal desorption spectra of the main desorbed gases in carbon films in the case of a 50 $\mathrm{mm}$ gap width. Deuterium retained in the film was mainly desorbed in the form of $\mathrm{D}_{2}, \mathrm{HD}, \mathrm{CD}_{4}$ and $\mathrm{C}_{2} \mathrm{D}_{4}$. The desorption spectra of $\mathrm{D}_{2}$ in the carbon film produced near the gap entrance (Fig. 5(a)) showed a broad peak at around 
(a)

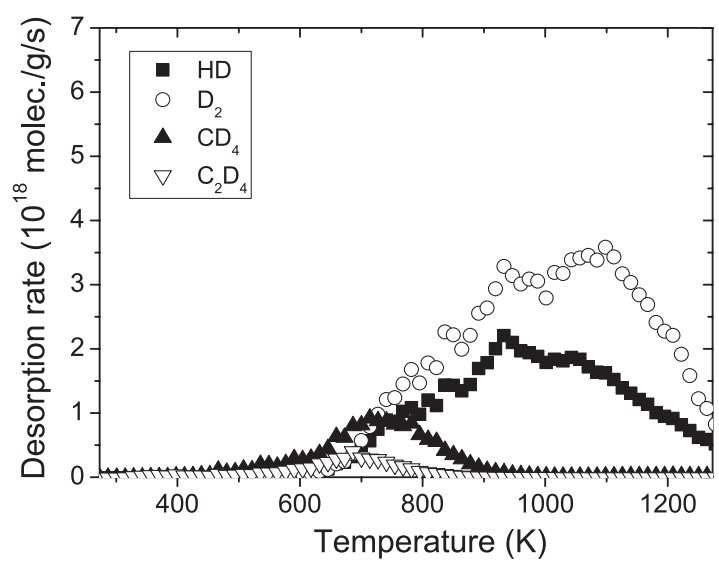

(b)

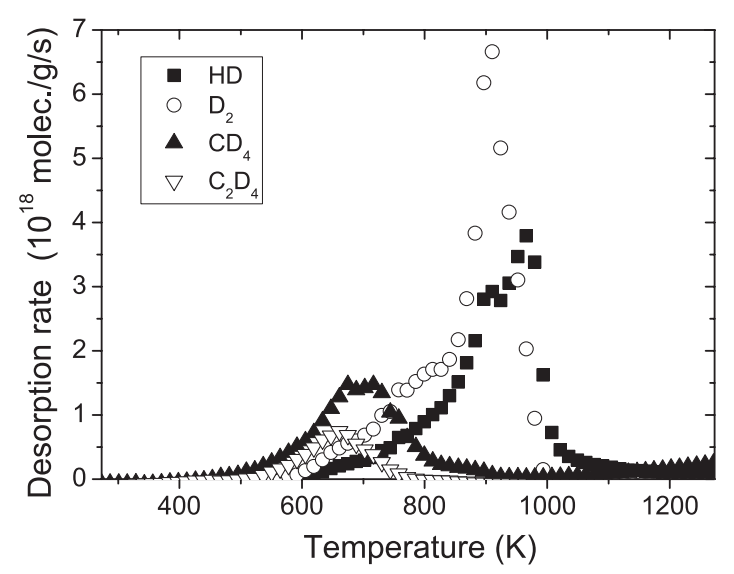

Fig. 5 Thermal desorption spectra of main desorbed gases for the film produced at the depth of $7 \mathrm{~mm}$ (a) and $82 \mathrm{~mm}$ (b) from gap entrance in the case of $50 \mathrm{~mm}$ gap width.

$1100 \mathrm{~K}$. This peak temperature was almost the same as the case of graphite irradiated by energetic hydrogen ions [12]. This suggests that the deuterium retained in the film was trapped by chemical bond with carbon atoms. Also, the desorption peaks in the carbon film produced near the entrance were broader when compared with the irradiation case. This would be caused by a wide variety of binding energy of deuterium with carbon atoms in the amorphous carbon film produced in this study and/or by differences in the depth at which the deuterium was trapped within the film. Compared to the carbon film near the gap entrance, the TDS spectra of $\mathrm{D}_{2}$ in the carbon film produced near the bottom (Fig. 5 (b)) showed much sharper peaks. As seen in Fig. 4 (b), the carbon film produced near the bottom in the gap had a more amorphous structure compared to the films produced near the gap entrance. More amorphized carbon films easily transforms from a disordered amorphous structure to a more graphitic structure when the films are annealed $[13,14]$. This transformation of the microstructure during the annealing in TDS, especially for the carbon film produced in deeper regions, might be responsible for its sharper D2 desorption peak.

\section{Summary}

In this study, carbon deposition behavior and deuterium desorption properties of co-deposited carbon film produced in gap with various gap widths were investigated. The dependence of the atomic ratio of $\mathrm{D} / \mathrm{C}$ on the distance from gap entrance differed for different gap widths cases. In the case of a wide gap, $\mathrm{D} / \mathrm{C}$ was almost constant in gap, while in the case of narrow gap $\mathrm{D} / \mathrm{C}$ decreased with increasing distance from the entrance. This would be caused by the decrease in flux ratio of deuterium atoms to carbon atoms in the deep region in the narrow gap case. The crystal structure of the carbon film became more amorphous in the deeper region in the gap, possibly owing to the deposition of low energy particles. Deuterium desorption properties depend on the depth where the film was produced. This might be due to differences in micro structure and/or in deuterium concentration $(\mathrm{D} / \mathrm{C})$. The results suggest that carbon deposition profile and deuterium retention properties of co-deposited carbon film in gap can be affected by the gap width and the depth at which the film is produced.

[1] G. Federici et al., Nucl. Fusion 41, 1967 (2001).

[2] I. Tanarro et al., J. Nucl. Mater. 390-391, 696 (2009).

[3] C. Hopf et al., J. Nucl. Mater. 363-365, 882 (2007).

[4] T.S. Selinger et al., J. Nucl. Mater. 390-391, 602 (2009).

[5] W. Jacob et al., J. Nucl. Mater. 337-339, 839 (2005).

[6] Y. Nobuta et al., J. Nucl. Mater. 417, 607 (2011).

[7] K. Niwase et al., J. Nucl. Mater. 191-194, 335 (1992).

[8] M. Yoshida et al., J. Nucl. Mater. 386-388, 841 (2009).

[9] G. Capote et al., Sci. Eng. B 112, 101 (2004).

[10] J.G. Buijnsters et al., Vacuum 81, 1412 (2007).

[11] C. Casiraghi et al., Diam. Relat. Mater. 14, 1098 (2005).

[12] Y. Yamauchi et al., J. Nucl. Mater. 241-243, 1016 (1997).

[13] G. Moriotto et al., Thin Solis Films 241, 255 (1994).

[14] J. Podder et al., Diam. Relat. Mater. 241, 255 (1994). 